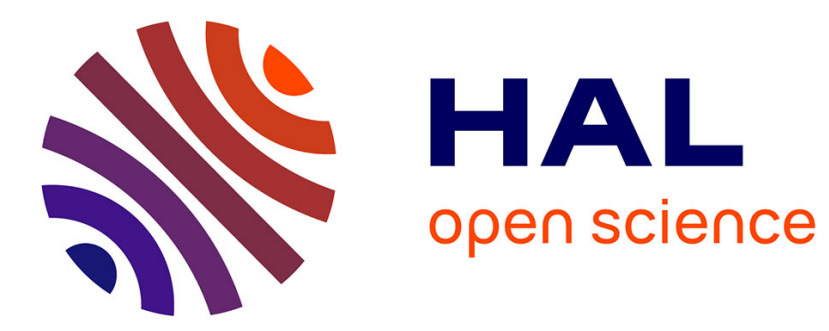

\title{
A semiclassical Thomas-Fermi model to tune the metallicity of electrodes in molecular simulations
}

Laura Scalfi, Thomas Dufils, Kyle G Reeves, Benjamin Rotenberg, Mathieu Salanne

\section{- To cite this version:}

Laura Scalfi, Thomas Dufils, Kyle G Reeves, Benjamin Rotenberg, Mathieu Salanne. A semiclassical Thomas-Fermi model to tune the metallicity of electrodes in molecular simulations. Journal of Chemical Physics, 2020, 153 (17), pp.174704. 10.1063/5.0028232 . hal-02988148

\section{HAL Id: hal-02988148 \\ https://hal.sorbonne-universite.fr/hal-02988148}

Submitted on 4 Nov 2020

HAL is a multi-disciplinary open access archive for the deposit and dissemination of scientific research documents, whether they are published or not. The documents may come from teaching and research institutions in France or abroad, or from public or private research centers.
L'archive ouverte pluridisciplinaire HAL, est destinée au dépôt et à la diffusion de documents scientifiques de niveau recherche, publiés ou non, émanant des établissements d'enseignement et de recherche français ou étrangers, des laboratoires publics ou privés. 


\section{A semiclassical Thomas-Fermi model to tune the metallicity of electrodes in molecular simulations $\odot$ ( $)$}

Cite as: J. Chem. Phys. 153, 174704 (2020); https://doi.org/10.1063/5.0028232

Submitted: 03 September 2020 . Accepted: 14 October 2020 . Published Online: 04 November 2020

(iD) Laura Scalfi, (D) Thomas Dufils, (D) Kyle G. Reeves, (D) Benjamin Rotenberg, and (D) Mathieu Salanne

\section{COLLECTIONS}

F This paper was selected as Featured

SCI This paper was selected as Scilight

\section{Meet the Next Generation of Quantum Analyzers And Join the Launch

Register now

Zurich

Instruments 


\title{
A semiclassical Thomas-Fermi model to tune the metallicity of electrodes in molecular simulations ${ }^{\circ}$ (ङ)
}

\author{
Cite as: J. Chem. Phys. 153, 174704 (2020); doi: 10.1063/5.0028232 \\ Submitted: 3 September 2020 - Accepted: 14 October 2020 • \\ Published Online: 4 November 2020
}

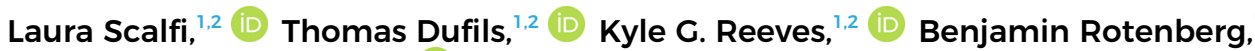 \\ and Mathieu Salanne ${ }^{1,2,3, a)}$ (D)
}

\begin{abstract}
AFFILIATIONS
'Sorbonne Université, CNRS, Physico-chimie des Électrolytes et Nanosystèmes Interfaciaux, PHENIX, F-75005 Paris, France ${ }^{2}$ Réseau sur le Stockage Electrochimique de l'Energie (RS2E), FR CNRS 3459, 80039 Amiens Cedex, France

${ }^{3}$ Institut Universitaire de France (IUF), 75231 Paris Cedex 05, France
\end{abstract}

a) Author to whom correspondence should be addressed: mathieu.salanne@sorbonne-universite.fr

\begin{abstract}
Spurred by the increasing needs in electrochemical energy storage devices, the electrode/electrolyte interface has received a lot of interest in recent years. Molecular dynamics simulations play a prominent role in this field since they provide a microscopic picture of the mechanisms involved. The current state-of-the-art consists of treating the electrode as a perfect conductor, precluding the possibility to analyze the effect of its metallicity on the interfacial properties. Here, we show that the Thomas-Fermi model provides a very convenient framework to account for the screening of the electric field at the interface and differentiating good metals such as gold from imperfect conductors such as graphite. All the interfacial properties are modified by screening within the metal: the capacitance decreases significantly and both the structure and dynamics of the adsorbed electrolyte are affected. The proposed model opens the door for quantitative predictions of the capacitive properties of materials for energy storage.
\end{abstract}

Published under license by AIP Publishing. https://doi.org/10.1063/5.0028232

\section{INTRODUCTION}

The development of constant applied potential methods for simulating electrochemical systems ${ }^{1}$ has allowed solving many outstanding problems in physical electrochemistry, ranging from the origin of supercapacitance in nanoporous electrodes made of carbon ${ }^{2}$ or even of metal organic frameworks to the understanding of the dynamic aspects of metal surface hydration. ${ }^{4}$ These methods are based on the use of an extended Hamiltonian in which the electrode charges are additional degrees of freedom that obey a constant potential constraint at each simulation step. ${ }^{5}$ They allowed to partly alleviate the main conceptual difficulty to represent the electrode-electrolyte interface at the molecular scale, which is the need to account for the electronic structure on the electrode side, while the electrolyte is usually better simulated using classical force fields because it requires a sampling of the configurational space beyond the reach of today's capabilities with $a b$ initio calculations (see Ref. 6 for a recent review of classical molecular simulations of electrode-electrolyte interfaces).

Despite these successes, the possibility to simulate realistic systems remains limited by the crudeness of the "electronic structure" model, since the electrode is treated as a perfect metal. It is, however, well known that the electronic response of different electrodes (e.g., graphite vs gold) to the adsorption of a charge should strongly differ. This was shown in numerous analytical ${ }^{7,8}$ or density functional theory (DFT)-based studies, ${ }^{9,10}$ but also more recently in an experimental study where strong differences in the confinement-induced freezing of ionic liquids were shown depending on the nature of the electrode. ${ }^{11}$ In the latter study, this effect was interpreted using analytical developments accounting for the metallicity of the system in the framework of the Thomas-Fermi (TF) model. ${ }^{1}$

Here, we build upon these developments to implement a computational Thomas-Fermi electrode. The TF model ${ }^{13,14}$ is based on 
a local density approximation of the free electron gas, limited to its kinetic energy, and it accounts for the screening of the electrostatic potential over a characteristic screening length. We consider model electrodes with the gold structure and tunable metallicity, separated by either vacuum or a simple $\mathrm{NaCl}$ aqueous electrolyte. We show that both the total accumulated charge and its distribution within the electrode are strongly affected. Accounting for screening in the electrodes radically changes their response to the adsorption of the electrolyte, which results in noticeable differences in the structure of the liquid when a voltage is applied. Screening inside the metal should therefore be accounted for when simulating electrochemical interfaces in applications ranging from supercapacitors to Li-ion batteries.

\section{THE THOMAS-FERMI ELECTRODE MODEL}

We consider an electrode composed of $N_{s}$ sites (here, these sites are positioned on the nuclei) with a number density $d$. Each atom $i$ has $Z$ valence electrons, and we introduce its partial charge $q_{i}$ as a dynamical variable accounting for the local excess of electrons. As shown schematically in Fig. 1, in the currently available method, the charges fluctuate in time to represent perfect metals. The partial charges are calculated at each simulation step in order to ensure that the potential is the same within the whole electrode; ${ }^{15}$ when such an electrode is put in contact with an electrolyte, the screening occurs within a thin layer at the surface only [note that supercapacitors are often simulated using constant charge setups in which the vector $\left\{q_{i}\right\}_{i \in\left[1, N_{s}\right]}$ contains prescribed (usually identical) values for all the atoms of each electrode and does not vary with time, which does not correspond to a realistic electrode]. Nevertheless, many electrode materials have a finite density of states available at the Fermi level. This was sometimes considered in the literature by computing the so-called quantum capacitance that accounts for the corresponding screening. ${ }^{10,16}$

Here, we propose taking these effects into account directly within classical molecular dynamics simulations by employing the Thomas-Fermi model. It consists of a local density approximation of the energy of the valence electrons. The Thomas-Fermi functional for the kinetic energy reads

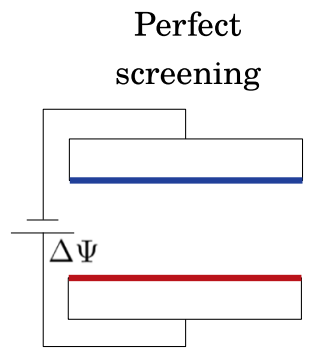

Ideal metal
Thomas-Fermi screening

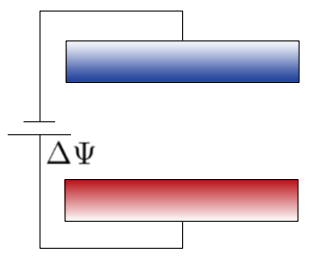

Non ideal metal
FIG. 1. Electrode polarization with different simulation methods. Constant potential simulations (left) correspond to a perfect screening of the charges, hence to the behavior of an ideal metal, whereas the Thomas-Fermi model introduces a screening length to account for the imperfect screening of the charge in a non-ideal metal.

$$
U_{T F}[n(\mathbf{r})]=\int \frac{3}{10} \frac{\hbar^{2}}{m_{e}}\left(3 \pi^{2}\right)^{2 / 3} n(\mathbf{r})^{5 / 3} \mathrm{~d} \mathbf{r}
$$

where $n(\mathbf{r})$ is the local number density of electrons, with $m_{e}$ being their mass, and $\hbar$ being Planck's constant. In order to obtain a practical description in molecular simulations, we now express $n(\mathbf{r})$ as a sum over discrete atomic sites $i$, with local densities $n_{i}=d\left[Z+\frac{q_{i}}{(-e)}\right]$, with $e$ being the elementary charge. If the perturbation in the number of free charge carriers is small compared to the number of electrons, i.e., $\left|q_{i}\right| \ll Z e$, we can expand the kinetic energy to second order in powers of $q_{i}$ as

$$
U_{T F}=\frac{3}{5} N_{s} Z E_{F}+\frac{E_{F}}{(-e)} \sum_{i=1}^{N_{s}} q_{i}+\frac{l_{T F}^{2} d}{2 \epsilon_{0}} \sum_{i=1}^{N_{s}} q_{i}^{2},
$$

where $E_{F}=\hbar^{2} k_{F}^{2} / 2 m_{e}$ is the Fermi level of a free-electron gas of density $Z d$ and $l_{T F}=\sqrt{\epsilon_{0} \hbar^{2} \pi^{2} /\left(m_{e} e^{2} k_{F}\right)}$ is the Thomas-Fermi length of the material, with the corresponding Fermi wavevector defined by $k_{F}^{3} / 3 \pi^{2}=Z d$ and $\epsilon_{0}$ being the vacuum permittivity. The zerothorder term is the total kinetic energy of an electron gas with $N_{s} Z$ electrons (the total number of electrons in the system). The first order corresponds, by definition, to the chemical potential of the added/removed electrons (depending on the sign of $q_{i}$ ). The second order term that is always positive and reaches its minimum when all the partial charges vanish corresponds to an energy penalty to induce non-homogeneous charge distributions.

Our system consists of two electrodes, hereafter named after their positions in the simulation cell: left $(\mathrm{L})$ and right $(\mathrm{R})$. Their atom indices range between $\left[1, N_{L}\right]$ and $\left[N_{L}+1, N_{L}+N_{R}\right]$, their Thomas-Fermi energies are denoted as $U_{T F}^{L}$ and $U_{T F}^{R}$, and they are held at potentials $\Psi_{L}$ and $\Psi_{R}=\Psi_{L}+\Delta \Psi$, respectively, where $\Delta \Psi$ is the applied voltage. We assume, for simplicity, that the electrodes are made of the same material, and hence, they have the same Fermi level at rest. The total energy of the system reads

$$
E_{\text {tot }}=K+U_{C}+U_{v d W}+U_{T F}^{L}+U_{T F}^{R}-\sum_{i=1}^{N_{L}} \Psi_{L} q_{i}-\sum_{i=N_{L}+1}^{N_{L}+N_{R}} \Psi_{R} q_{i}
$$

where $K$ is the kinetic energy of the electrolyte, $U_{C}$ corresponds to the Coulombic interactions, and $U_{v d W}$ describes the van der Waals interactions (given by a force field), while the last two terms account for the reversible work necessary to charge the electrode atoms. $U_{C}$ reads

$$
U_{C}=\frac{1}{2} \iint \frac{\rho(\mathbf{r}) \rho\left(\mathbf{r}^{\prime}\right)}{4 \pi \epsilon_{0}\left|\mathbf{r}-\mathbf{r}^{\prime}\right|} \mathrm{d} \mathbf{r} \mathrm{d} \mathbf{r}^{\prime},
$$

where the charge distribution $\rho(\mathbf{r})$ consists of a collection of $M$ point charges for the electrolyte and $N=N_{L}+N_{R}$ atom-centered Gaussians (with width $\eta^{-1}$ ) representing the electrodes,

$$
\rho(\mathbf{r})=\sum_{j=1}^{M} q_{j} \delta\left(\mathbf{r}-\mathbf{r}_{j}\right)+\sum_{i=1}^{N} q_{i} \eta^{3} \pi^{-3 / 2} e^{-\eta^{2}\left|\mathbf{r}-\mathbf{r}_{i}\right|^{2}},
$$

with $\delta$ being the Dirac function. Note that in Eq. (4), the only selfenergy to be included is the one due to the Gaussian charges. For an electrochemical cell in which the two electrodes are made of the 
same material (hence, $E_{F}$ and $l_{T F}$ are equal), by injecting Eq. (2) into Eq. (3) and introducing $\Delta \Psi$, the total energy can be rewritten as

$$
E_{\mathrm{tot}}=K+U_{C}+U_{v d W}+\frac{3}{5} N Z E_{F}+\frac{l_{T F}^{2} d}{2 \epsilon_{0}} \sum_{i=1}^{N} q_{i}^{2}-\Delta \Psi Q_{t o t},
$$

where we imposed the electroneutrality constraint $\sum_{i=1}^{N} q_{i}=0$, as detailed in Ref. 5, so that the electrodes bear opposite charges and the corresponding term in the reversible work reduces to the usual $Q_{\text {tot }} \Delta \Psi$, with $Q_{\text {tot }}$ being the total charge of the positive electrode. As in the constant potential method neglecting the quantum nature of the electrons (corresponding to $l_{T F}=0.0 \AA$ ), the charges are treated as dynamic variables, which are obtained at each time step of the simulation by enforcing the constant potential constraint $\partial E_{\text {tot }} / \partial q_{i}$ $=0 .{ }^{5,15}$ Compared to this perfect metal case, the modifications of the algorithm are minimal and virtually do not add any computational cost.

Our approach, which involves fluctuating charges, may be related to the charge equilibration model, ${ }^{17-19}$ particularly to its extension to electrochemical systems proposed by Onofrio et al. ${ }^{20}$ This method is based on two main chemical quantities, the electronegativity $\chi$ and the hardness $H$ of each atomic species. The self-consistent equations to solve are equivalent if we take $\chi \sim E_{F}$ and $H \sim e^{2} l_{T F}^{2} d / \epsilon_{0}$. However, these concepts, which are related to those of electronic affinity and ionization energy, ${ }^{21}$ are rooted in the description of the electronic properties of atoms and molecules, rather than that of bulk materials, which are more naturally described in terms of the band structure. The issue of starting from the correct reference state for (electro-)chemical potential equalization methods was already pointed out in Ref. 22, where York and Yang derived a fluctuating charge model from DFT for molecules and underlined the difference between atomic and molecular reference states to determine the electronegativities and hardnesses. More recently, a detailed discussion on the correspondence between constant potential electrode models and the charge equilibration approach was provided in Ref. 23. Another physical model of electrodes was proposed ${ }^{24}$ in which the Hamiltonian is constructed in the tight-binding approximation.

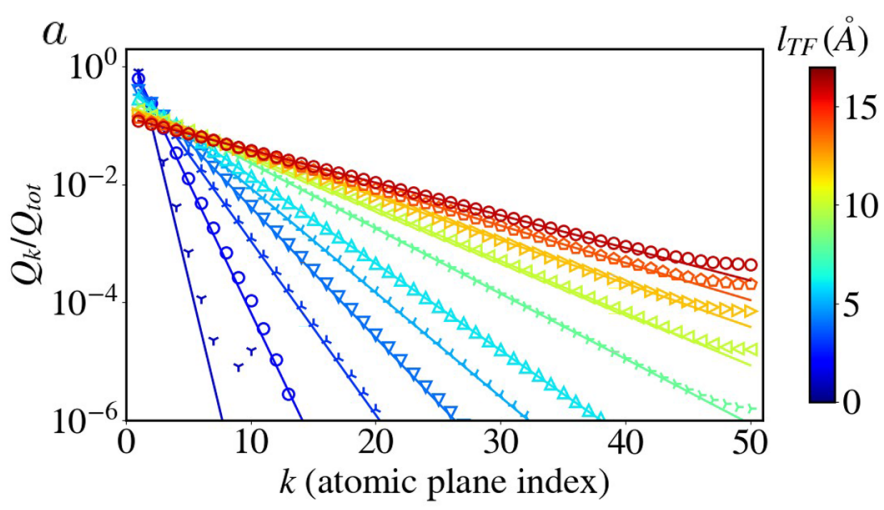

C

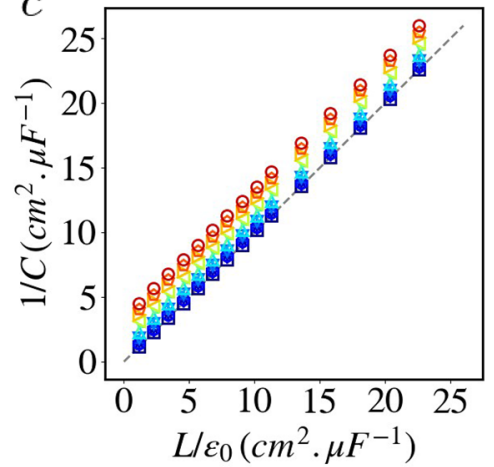

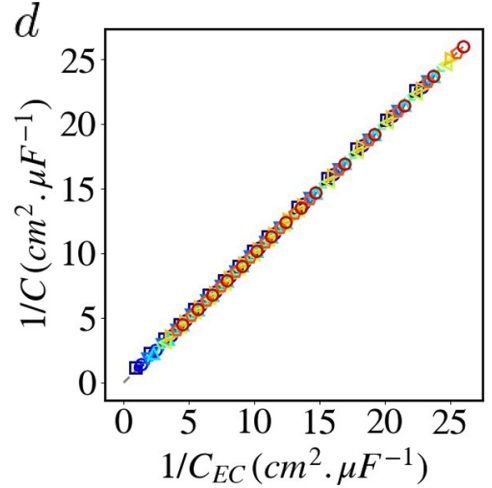
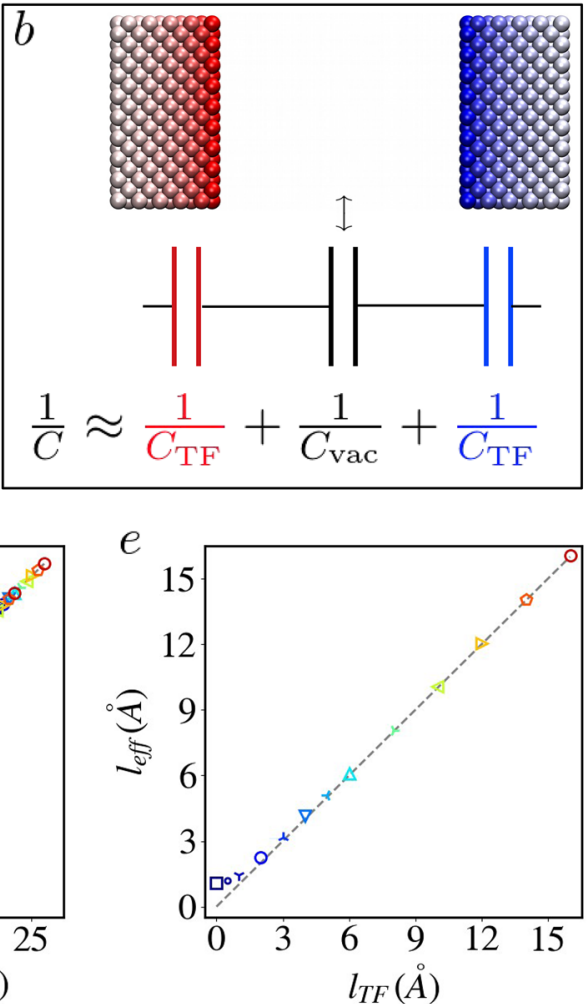

FIG. 2. Empty Thomas-Fermi capacitor. All results correspond to a (100) gold-like electrode structure with $n=50$ atomic planes and $L=300 \AA$ between the electrodes where not stated otherwise. Charges are computed by applying a voltage $\Delta \Psi=1 \mathrm{~V}$ between the electrodes for different Thomas-Fermi lengths $I_{T F}$ ranging from $0.0 \AA$ to $16.0 \AA$, which are represented both by different symbols and by different colors indicated by the color bar. (a) Total charge per plane on the positive electrode as a function of the position from the surface ( $k$ is the index of the atomic plane), normalized by the total electrode charge $Q_{\text {tot }}$ (only values for $I_{T F}>0.5 \AA$ are shown). The symbols are simulated values for different Thomas-Fermi lengths $I_{T F}$, and the lines are the prediction of Eq. (7). (b) Snapshot of the simulated system and its equivalent circuit representation corresponding to the capacitance obtained with the continuum theory (see the text). (c) Computed reciprocal capacitance as a function of the analytical predictions for perfect metals using $L_{v a c}=L$ and (d) for Thomas-Fermi metals using Eq. (8) with $L_{v a c}=L-a$ for varying electrode spacing $L$ (between $10 \AA$ and $200 \AA$ ). (e) Effective length $I_{\text {eff }}$, defined in Eq. (9), as a function of $I_{T F}$. 


\section{EMPTY CAPACITOR}

As a first validation of our implementation, we study a model system composed of two planar (100) gold electrodes separated by a distance $L$ and held at a constant potential difference $\Delta \Psi=1 \mathrm{~V}$. Each electrode consists of $n$ atomic planes with an inter-spacing $a$ in the $z$ direction. We compare the simulated results against analytical predictions of the corresponding continuum model where the Poisson equation for the one-dimensional potential $\Psi(z)$ reads $\Psi^{\prime \prime}(z)=l_{T F}^{-2} \Psi(z)$ inside each electrode and $\Psi^{\prime \prime}(z)=0$ between them. The total capacitance of the system is given by $C=Q_{t o t} / \Delta \Psi$.

Assuming that the width of the material is large compared to the Thomas-Fermi length, the in-plane charge $Q_{k}$ at $z=k a(k \in[1$, $n]$ ) can be expressed as

$$
\frac{Q_{k}}{Q_{\text {tot }}}=e^{-(k-1) a / l_{T F}}\left[1-e^{-a / l_{T F}}\right] .
$$

Figure 2(a) shows a very good agreement between Eq. (7) and the simulation for large $l_{T F}$ values. Small deviations are observed for large $z$ due to the finite number of planes and for $l_{T F}$ values smaller than characteristic atomic lengths where the continuum prediction is not expected to hold. The above exponentially decaying charge distribution inside the metal, due to the screening over the ThomasFermi length $l_{T F}$, results according to the continuous model in a capacitance per unit area,

$$
\frac{1}{C_{E C}}=\frac{1}{C_{v a c}}+\frac{2}{C_{T F}}=\frac{L_{v a c}}{\epsilon_{0}}+\frac{2 l_{T F}}{\epsilon_{0}},
$$

with $C_{v a c}=\epsilon_{0} / L_{v a c}$ being the theoretical capacitance per unit area for perfect metallic electrodes $\left(l_{T F}=0.0 \AA\right)$ separated by a vacuum slab of width $L_{v a c}$ and $C_{T F}=\epsilon_{0} / l_{T F}$ being that for a single Thomas-Fermi electrode. This result can be simply understood in terms of the equivalent circuit (hence the subscript $C_{E C}$ ) illustrated in Fig. 2(b), with three capacitors in series (see Sec. S1 of the supplementary material for a discussion of the continuum descriptions and equivalent circuit models). As shown in Fig. 2(c), the simulation results are consistent with the prediction of a linear relation between $1 / C$ and $L / \epsilon_{0}$, where $L$ is the distance between the first atomic planes on each electrode, with a constant shift that increases with $l_{T F}$.

However, the width of the vacuum slab between the electrodes is not exactly the distance between the first atomic planes. Indeed, each atomic site is surrounded by electrons, and the boundary between the free electron gas inside the electrode and the vacuum $^{25}$ (the so-called "Jellium edge" ${ }^{26}$ ) is rather shifted half of the inter-plane distance away from the electrode. Since this feature is present on both electrodes, the actual vacuum slab width is more consistent with $L_{v a c}=L-a$. Figure 2 (d) shows that using this prescription, Eq. (8) provides a very good description of the simulated capacitance $C$ over a wide range of distances between the electrodes and Thomas-Fermi lengths, which confirms the consistency of the present classical model to represent the charge distribution within the metal. The decay length of the charge inside the electrode coincides with $l_{T F}$ within $1 \%$ for all values $l_{T F} \gtrsim a$. The slight deviations from the predictions of the continuous theory can be analyzed by introducing an effective length $l_{\text {eff }}$ from the measured capacitance as

$$
\frac{1}{C}=\frac{L-a}{\epsilon_{0}}+\frac{2 l_{e f f}}{\epsilon_{0}} .
$$

The results obtained for various $l_{T F}$ at fixed $L$, illustrated in Fig. 2(e), indicate that this effective length deviates from the Thomas-Fermi length only when the latter becomes comparable to the atomic details of the electrodes (interplane and interatomic distances, width of the Gaussian distributions). An additional test was performed by adding a single charge at various distances between the electrodes and comparing the energy of the system to an approximate analytical expression. ${ }^{12}$ The results, which are provided in Sec. S2 of the supplementary material, also show a good agreement over a broad range of $l_{T F}$ values.

\section{IMPACT OF THE THOMAS-FERMI LENGTH ON THE ELECTROCHEMICAL INTERFACE PROPERTIES}

In order to understand the impact of screening inside the metal on the properties of electrode/electrolyte interfaces, we study
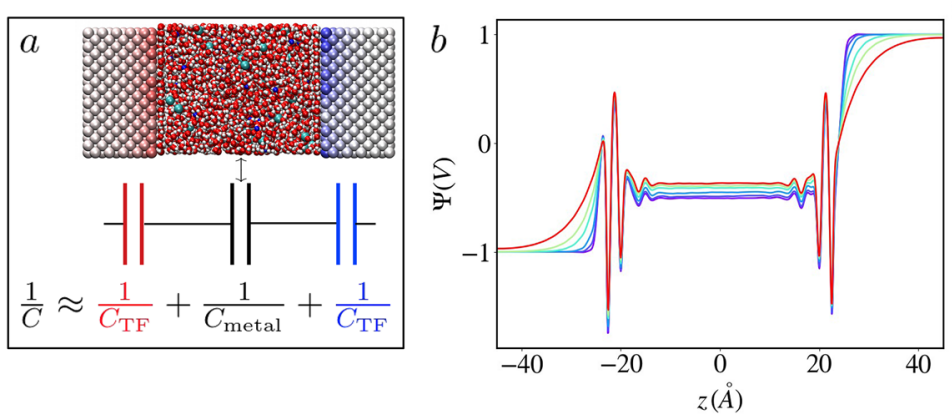

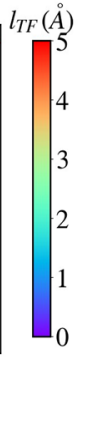

FIG. 3. The capacitance decreases significantly with the Thomas-Fermi length. (a) Snapshot of the simulated system and its equivalent circuit representation, where $C_{\text {metal }}$ stands for the capacitance computed for the perfect metal simulation. (b) Poisson potential across the simulation cell for a system made of two (100) gold-like electrodes in

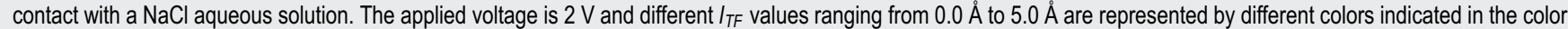
bar. The screening of the potential inside the electrodes increases markedly with $I_{T F}$. (c) Variation of the capacitance with $I_{T F}$. The results from the simulations are compared with the equivalent circuit approximation. Error bars are extracted from the standard error of the charge distribution corrected for sample correlations. 
a system consisting of two (100) gold-like electrodes in contact with an aqueous solution of $\mathrm{NaCl}$ (with concentration $1 \mathrm{~mol} \mathrm{l}^{-1}$ ), illustrated in Fig. 3(a). The TF length $l_{T F}$ was systematically varied from $0.0 \AA$ to $5.0 \AA$ in order to switch from a perfect metal to typical semi-metallic conditions (estimations yield typical values of $0.5 \AA$ for platinum, $1.5 \AA$ for doped silicon, and $3.4 \AA$ for graphite $^{11}$ ). Simulations were performed for voltages $\Delta \Psi=0 \mathrm{~V}, 1 \mathrm{~V}$, and $2 \mathrm{~V}$ between the two electrodes.

As a first illustration of the impact of screening on the electrochemical interface, we compute the Poisson potential across the cell.
The results for an applied potential of $2 \mathrm{~V}$ are displayed on Fig. 3(b). We observe a very different pattern inside the electrode depending on $l_{T F}$ : for the perfect metal, the applied potential is reached at positions corresponding to the first atomic plane, while for the TF model, we clearly see the desired effect of field penetration with an exponential decay inside the electrode. Note also that at the largest applied voltage, the average atomic charge ranges between $0.02 e$ for $l_{T F}=0 \AA$ and $0.003 e$ for $l_{T F}=5 \AA$ (with corresponding standard deviations of $0.01 e$ and $0.001 e$ ), which validates a posteriori the hypothesis on the number of free charge carriers being smaller than the
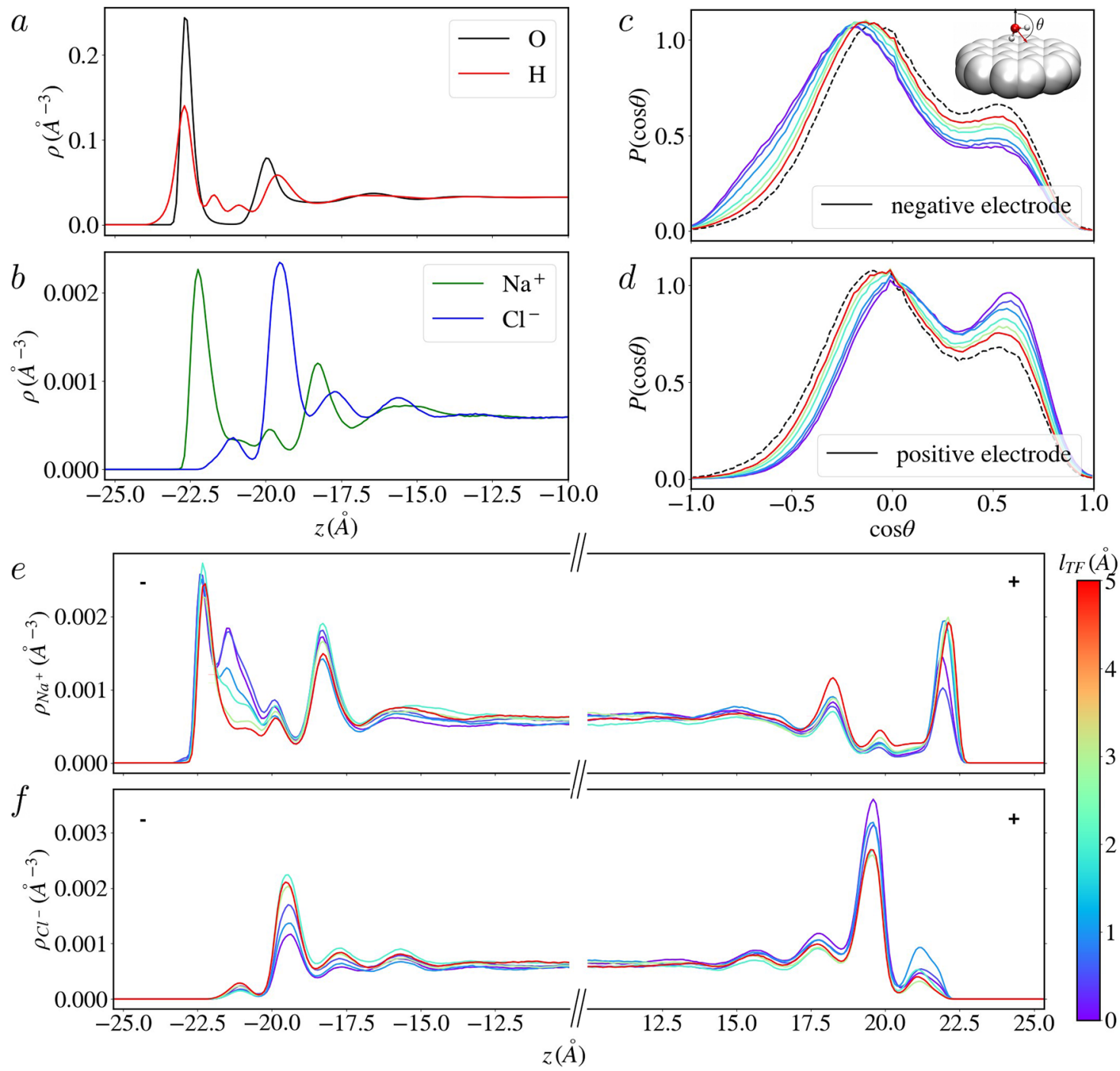

FIG. 4. The structure of the electrochemical interface depends on the Thomas-Fermi length at finite voltages. [(a) and (b)] Atomic density profiles for the $\mathrm{O}, \mathrm{H} \mathrm{Na}^{+}$, and $\mathrm{Cl}^{-}$ atoms near the electrode at null potential for $I_{T F}=0.0 \AA$ (the profiles are the same for the other $I_{T F}$ values, as shown in Fig. S 3 of the supplementary material). Note that in the case of $\mathrm{H}$ atoms, the profile is divided by two to facilitate the comparison with $\mathrm{O}$ atoms. [(c) and (d)] Distribution of the orientation of adsorbed water molecules with respect to the vector normal to the electrode surfaces for an applied potential of $2 \mathrm{~V}$ for the whole range of simulated $I_{T F}$ indicated by the color bar; the distribution for $0 \mathrm{~V}$ and $I_{T F}$ $=0.0 \AA$ is also reported (black dashed lines) as a reference. [(e) and (f)] Atomic density profiles for the $\mathrm{Na}^{+}$and $\mathrm{Cl}^{-}$ions for an applied potential of $2 \mathrm{~V}$ for the whole range of simulated $I_{T F}$ indicated by the color bar. The negative (positive) electrode is located at negative (positive) $z$. 
number of electrons made in the derivation of the TF electrode model.

Figure 3(c) shows that the integral capacitance decreases significantly with $l_{T F}$ (note that it remains constant between $1 \mathrm{~V}$ and $2 \mathrm{~V}$ and that the decrease is of similar magnitude for the individual capacitance of the positive and negative electrodes; see Fig. S2 of the supplementary material). The effect is already non-negligible for $l_{T F}=0.5 \AA$ (which is representative of many real metals) since the capacitance is $7 \%$ smaller than the one of the perfect metal; it is even more pronounced in the semi-metallic régime. This can be understood by noting that the TF length varies as the inverse square-root of the number of available states at the Fermi level. In a perfect metal, the number of accessible states is infinite so that the only resistance to charging arises from the Coulombic energy. In contrast, the TF model results in an additional energy penalty for increasing the surface charge, described by the quadratic term in Eq. (6).

As for the empty capacitor, it is possible to estimate the capacitance from the value for the perfect metal $C_{\text {metal }}$ using the equivalent circuit depicted on Fig. 3(a) (see Sec. S1 of the supplementary material). This approach, used by Gerischer to interpret experimental data, ${ }^{27}$ has been applied in many simulation works where the additional term due to the screening was computed using DFT and therefore called "quantum capacitance," while the perfect metal capacitance was computed using either a mean-field theory ${ }^{10}$ or molecular dynamics. ${ }^{28}$ Nevertheless, it neglects the interplay between the electronic structure of the electrode and the ionic structure of the adsorbed electrolyte. This coupling is self-consistently taken into account in our model, which therefore provides a perfect framework to test this approximation. As shown in Fig. 3(c), the equivalent circuit approximation underestimates rather significantly the real capacitance (by $20 \%-30 \%$ ).

At null voltage, the average structure of the liquid does not vary significantly with $l_{T F}$ (see Fig. S3 of the supplementary material). As shown in Figs. 4(a) and 4(b), it is characterized by several adsorption layers, mainly consisting of water molecules. By computing the distribution of the angle $\theta$ between the vector normal to the surface and the water dipole [see the black dashed curve in Figs. 4(c) and 4(d)] or the $\mathrm{O}-\mathrm{H}$ bonds (see Fig. S4 of the supplementary material) for molecules in the first adsorbed layer, we observe that they mostly lie in a plane parallel to the surface or with one $\mathrm{H}$ atom pointing away from the surface. A small population is oriented toward the surface, which results in a small shoulder on atomic density profiles of the $\mathrm{H}$ atoms.

The ions have different adsorption profiles: the $\mathrm{Na}^{+}$density is characterized by a large peak located close to one of the $\mathrm{O}$ atoms so that they can be considered to belong to the first layer, while the $\mathrm{Cl}^{-}$ ions are located further away from the electrode surface. Their profile displays a small pre-peak in the region where the water density is very low and a peak with a larger intensity located in the second hydration layer. Once a potential is applied, the liquid mainly responds to the two electrodes through (i) a stronger orientation of the water molecules toward/away from the negative/positive electrode, as shown in Figs. 4(c) and 4(d) and Figs. S4 and S5 of the supplementary material, and (ii) the appearance of a new adsorption peak for the $\mathrm{Na}^{+}$ions near the negative electrode [Fig. 4(e)] and an increase in the pre-peak intensity in the $\mathrm{Cl}^{-}$density profiles on the positive electrode side [Fig. 4(f)]. In all cases, the modifications in

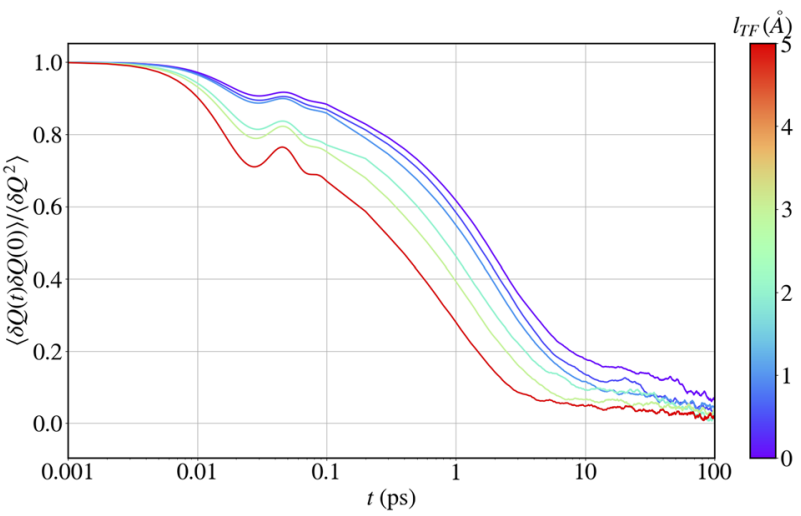

FIG. 5. The relaxation of the electrode charge indicates a faster dynamics of the interfacial electrolyte near screened metals. Normalized auto-correlation function of the total charge at null potential for varying I IF values ranging from $0.0 \AA$ to $5.0 \AA$ indicated by the color bar.

the structure depend strongly on $l_{T F}$. This shows that depending on the type of material, we can expect all the electrochemical doublelayer properties to change markedly with the nature of the chosen electrode.

Dynamical properties are particularly important for electrochemical applications. They control the power delivered by an energy storage device. The equilibrium fluctuations of the electrode charge at $0 \mathrm{~V}$, which reflect the linear response to a small applied voltage, are shown in Fig. 5 for various $l_{T F}$. An increased screening yields faster dynamics for the relaxation of the electrochemical double-layer. Such a difference was somewhat unexpected given that the systems at null potential have, on average, the same structural features, but it can be qualitatively understood as the result of weaker interactions with the more diffuse charges induced within the electrode. This means that the dynamics do not only depend on the nature of the electrolyte but also depend on the electronic structure of the electrode material.

\section{CONCLUSION}

Understanding the electrode/electrolyte interface is a prerequisite not only for the design of more efficient energy storage devices ${ }^{29}$ but also for understanding wetting phenomena involved in lubrication or heterogeneous catalysis. ${ }^{30}$ Although in the past decades, molecular simulations have provided much insight into the structure of the electrochemical double-layer, they still fail at predicting quantitatively many experimental quantities, such as the variation of the differential capacitance with the applied voltage. ${ }^{31}$ This is particularly true in the case of carbon materials due to their complex electronic structure properties that deviate largely from the ones of typical metals. Many intriguing experimental observations, such as the capillary freezing of ionic liquids confined between metallic surfaces ${ }^{11}$ and the emergence of longer-than-expected electrostatic screening lengths in concentrated electrolytes, ${ }^{32,33}$ remain to be explained quantitatively. The Thomas-Fermi model, by allowing to tune the metallicity of the electrode using a single parameter (also without introducing additional computational costs), should lead to 
a more accurate understanding of the interfacial properties of such electrodes using molecular simulations. The extension of this work to complex materials such as nanoporous carbons will require additional efforts in order to take into account the effect of the local environment of each atom on its electronic response. In that case, it might be relevant to sacrifice some of the simplicity of the TF model by including atom-specific or even bond-specific terms in the energy, following the split charge equilibration approach. ${ }^{34,35}$ In this context, the present work suggests that it could be possible to determine the associated parameters from a simplified representation of the underlying electronic density.

\section{SUPPLEMENTARY MATERIAL}

See the Supplementary material for a discussion on the continuum description and equivalent circuit models, additional tests for a single charge between two electrodes, additional results on the differential capacitances of the two electrodes and their variation with the Thomas-Fermi length, and additional structural characterizations of the aqueous $\mathrm{NaCl}$ electrolyte put in contact with the gold-like electrodes at $0 \mathrm{~V}$ and $2 \mathrm{~V}$.

\section{AUTHORS' CONTRIBUTIONS}

L.S. and T.D. contributed equally to this work.

\section{ACKNOWLEDGMENTS}

The authors thank M. Sprik, P. A. Madden, L. Bocquet, and B. Coasne for useful discussions. This project received funding from the European Research Council under the European Union's Horizon 2020 research and innovation program (Grant Agreement No. 771294). This work was supported by the French National Research Agency [Labex STORE-EX (Grant No. ANR10-LABX-0076), project SELFIE (Grant No. ANR-17-ERC2-0028), and project NEPTUNE (Grant No. ANR-17-CE09-0046-02)]. The authors acknowledge HPC resources granted by GENCI (resources of CINES; Grant No. A0070911054).

\section{APPENDIX: SIMULATION DETAILS}

The TF electrode model was implemented in the molecular dynamics code MetalWalls. ${ }^{36}$ All simulations were run using a matrix inversion method ${ }^{5}$ to enforce both the constant potential and the electroneutrality constraints on the charges. Electrode atoms have a Gaussian charge distribution of width $\eta^{-1}=0.56 \AA$ centered on zero, and the Thomas-Fermi length $l_{T F}$ ranges from $0.0 \AA$ to $16.0 \AA$ for the empty capacitor and from $0.0 \AA$ to $5.0 \AA$ in the presence of an aqueous $\mathrm{NaCl}$ electrolyte. Two-dimensional boundary conditions were used with no periodicity in the $z$ direction using an accurate 2D Ewald summation method to compute electrostatic interactions. A cutoff of $17.0 \AA$ was used for both the short range part of the Coulomb interactions and the intermolecular interactions. For the latter, we used the truncated shifted LennardJones potential. The box length in both the $x$ and $y$ directions was $L_{x}=L_{y}=36.630 \AA$ with 162 atoms per atomic plane. The structure is face-centered cubic with a lattice parameter of $4.07 \AA$ and a separation between planes $a=2.035 \AA$ in the (100) direction (the atomic density $d$ is $0.59 \cdot 10^{29} \mathrm{~m}^{-3}$ ). The empty capacitors have 50 planes per electrode, whereas the electrochemical cells have 10 (leading to a total of 1620 atoms per electrode). In the latter case, the electrolyte is composed of 2160 water molecules, modeled using the SPC/E force field, ${ }^{37}$ and $39 \mathrm{NaCl}$ ion pairs. The Lennard-Jones parameters for $\mathrm{Na}^{+}$and $\mathrm{Cl}^{-}$were taken from Ref. 38 and the ones for the electrode atoms from Ref. 39; the Lorentz-Berthelot mixing rules were used. The simulation boxes were equilibrated at a constant atmospheric pressure for 500 ps by applying a constant pressure force to the electrodes with $l_{T F}=0.0 \AA$, and then, the electrode separation was fixed to the equilibrium value (for which the density in the middle of the liquid slab is equal to its bulk value) $L=56.8 \AA$. The simulations were run at $298 \mathrm{~K}$ with a time step of $1 \mathrm{fs}$. Each system was run for at least 8 ns.

\section{DATA AVAILABILITY}

The code used for the simulations and the data that support the findings of this study are openly available in the repository https://gitlab.com/ampere2.

\section{REFERENCES}

${ }^{1}$ J. I. Siepmann and M. Sprik, "Influence of surface-topology and electrostatic potential on water electrode systems,” J. Chem. Phys. 102, 511-524 (1995).

${ }^{2}$ C. Merlet, B. Rotenberg, P. A. Madden, P.-L. Taberna, P. Simon, Y. Gogotsi, and M. Salanne, "On the molecular origin of supercapacitance in nanoporous carbon electrodes," Nat. Mater. 11, 306-310 (2012).

${ }^{3}$ S. Bi, H. Banda, M. Chen, L. Niu, M. Chen, T. Wu, J. Wang, R. Wang, J. Feng, T. Chen, M. Dincă, A. A. Kornyshev, and G. Feng, "Molecular understanding of charge storage and charging dynamics in supercapacitors with MOF electrodes and ionic liquid electrolytes," Nat. Mater. 19, 552-558 (2020).

${ }^{4}$ D. T. Limmer, A. P. Willard, P. Madden, and D. Chandler, "Hydration of metal surfaces can be dynamically heterogeneous and hydrophobic," Proc. Natl. Acad. Sci. U. S. A. 110, 4200-4205 (2013).

${ }^{5}$ L. Scalfi, D. T. Limmer, A. Coretti, S. Bonella, P. A. Madden, M. Salanne, and B. Rotenberg, "Charge fluctuations from molecular simulations in the constantpotential ensemble,” Phys. Chem. Chem. Phys. 22, 10480-10489 (2020).

${ }^{6}$ L. Scalfi, M. Salanne, and B. Rotenberg, "Molecular simulation of electrodesolution interfaces," arXiv:2008.11967 (2020).

${ }^{7}$ A. A. Kornyshev and M. A. Vorotyntsev, "Analytic expression for the potential energy of a test charge bounded by solid state plasma," J. Phys. C: Solid State Phys. 11, L691-L694 (1978)

${ }^{8}$ A. A. Kornyshev, W. Schmickler, and M. A. Vorotyntsev, "Nonlocal electrostatic approach to the problem of a double layer at a metal-electrolyte interface," Phys. Rev. B 25, 5244-5256 (1982).

${ }^{9}$ N. B. Luque and W. Schmickler, “The electric double layer on graphite," Electrochim. Acta 71, 82-85 (2012).

${ }^{10}$ A. A. Kornyshev, N. B. Luque, and W. Schmickler, "Differential capacitance of ionic liquid interface with graphite: The story of two double layers," J. Solid State Electrochem. 18, 1345-1349 (2014).

${ }^{11}$ J. Comtet, A. Niguès, V. Kaiser, B. Coasne, L. Bocquet, and A. Siria, "Nanoscale capillary freezing of ionic liquids confined between metallic interfaces and the role of electronic screening," Nat. Mater. 16, 634-639 (2017).

${ }^{12}$ V. Kaiser, J. Comtet, A. Niguès, A. Siria, B. Coasne, and L. Bocquet, "Electrostatic interactions between ions near Thomas-Fermi substrates and the surface energy of ionic crystal at imperfect metals," Faraday Discuss. 199, 129-158 (2017).

${ }^{13}$ L. H. Thomas, “The calculation of atomic fields," Math. Proc. Cambridge Philos. Soc. 23, 542-548 (1927). 
${ }^{14}$ E. Fermi, "Un metodo statistico per la determinazione di alcune proprietà dell'atomo," Rend. Accad. Naz. Lincei 6, 602-607 (1927).

${ }^{15}$ S. K. Reed, O. J. Lanning, and P. A. Madden, "Electrochemical interface between an ionic liquid and a model metallic electrode," J. Chem. Phys. 126, 084704 (2007).

${ }^{16}$ E. Paek, A. J. Pak, and G. S. Hwang, "On the influence of polarization effects in predicting the interfacial structure and capacitance of graphene-like electrodes in ionic liquids," J. Chem. Phys. 142, 024701 (2015).

${ }^{17}$ R. F. Nalewajski, "Electrostatic effects in interactions between hard (soft) acids and bases," J. Am. Chem. Soc. 106, 944-945 (1984).

${ }^{18}$ W. J. Mortier, S. K. Ghosh, and S. Shankar, "Electronegativity-equalization method for the calculation of atomic charges in molecules," J. Am. Chem. Soc. 108, 4315-4320 (1986).

${ }^{19}$ A. K. Rappe and W. A. Goddard III, "Charge equilibration for molecular dynamics simulations," J. Phys. Chem. 95, 3358-3363 (1991).

${ }^{20}$ N. Onofrio, D. Guzman, and A. Strachan, "Atomic origin of ultrafast resistance switching in nanoscale electrometallization cells," Nat. Mater. 14, 440-446 (2015).

${ }^{21}$ M. Buraschi, S. Sansotta, and D. Zahn, "Polarization effects in dynamic interfaces of platinum electrodes and ionic liquid phases: A molecular dynamics study," J. Phys. Chem. C 124, 2002-2007 (2020).

${ }^{22}$ D. M. York and W. Yang, "A chemical potential equalization method for molecular simulations," J. Chem. Phys. 104, 159-172 (1996).

${ }^{23} \mathrm{H}$. Nakano and H. Sato, "A chemical potential equalization approach to constant potential polarizable electrodes for electrochemical-cell simulations," J. Chem. Phys. 151, 164123 (2019).

${ }^{24}$ L. Pastewka, T. T. Järvi, L. Mayrhofer, and M. Moseler, "Charge-transfer model for carbonaceous electrodes in polar environments," Phys. Rev. B 83, 165418 (2011).

${ }^{25} \mathrm{~N}$. D. Lang and W. Kohn, "Theory of metal surfaces: Induced surface charge and image potential," Phys. Rev. B 7, 3541-3550 (1973).

${ }^{26} \mathrm{~N}$. V. Smith, C. T. Chen, and M. Weinert, "Distance of the image plane from metal surfaces,” Phys. Rev. B 40, 7565-7573 (1989).
${ }^{27} \mathrm{H}$. Gerischer, "An interpretation of the double layer capacity of graphite electrodes in relation to the density of states at the Fermi level," J. Phys. Chem. 89, 4249-4251 (1985).

${ }^{28}$ A. J. Pak, E. Paek, and G. S. Hwang, "Relative contributions of quantum and double layer capacitance toward the supercapacitor performance of carbon nanotubes in an ionic liquid," Phys. Chem. Chem. Phys. 15, 19741-19747 (2013). ${ }^{29}$ M. Salanne, B. Rotenberg, K. Naoi, K. Kaneko, P.-L. Taberna, C. P. Grey, B. Dunn, and P. Simon, "Efficient storage mechanisms for building better supercapacitors," Nat. Energy 1, 16070 (2016).

${ }^{30} \mathrm{~J}$. Carrasco, A. Hodgson, and A. Michaelides, "A molecular perspective of water at metal interfaces," Nat. Mater. 11, 667-674 (2012).

${ }^{31}$ M. V. Fedorov and A. A. Kornyshev, "Ionic liquids at electrified interfaces," Chem. Rev. 114, 2978-3036 (2014).

${ }^{32}$ M. A. Gebbie, M. Valtiner, X. Banquy, E. T. Fox, W. A. Henderson, and J. N. Israelachvili, "Ionic liquids behave as dilute electrolyte solutions," Proc. Natl. Acad. Sci. U. S. A. 110, 9674-9679 (2013).

${ }^{33}$ A. M. Smith, A. A. Lee, and S. Perkin, "The electrostatic screening length in concentrated electrolytes increases with concentration," J. Phys. Chem. Lett. 7, 2157-2163 (2016).

${ }^{34}$ R. A. Nistor, J. G. Polihronov, and M. H. Müser, "A generalization of the charge equilibration method for nonmetallic materials," J. Chem. Phys. 125, 094108 (2006).

${ }^{35}$ R. A. Nistor and M. H. Müser, "Dielectric properties of solids in the regular and split-charge equilibration formalisms," Phys. Rev. B 79, 104303 (2009).

${ }^{36}$ A. Marin-Laflèche, M. Haefele, L. Scalfi, A. Coretti, T. Dufils, G. Jeanmairet, S. Reed, A. Serva, R. Berthin, C. Bacon, S. Bonella, B. Rotenberg, P. A. Madden, and M. Salanne, "Metalwalls: A classical molecular dynamics software dedicated to the simulation of electrochemical systems," J. Open Source Software 5, 2373 (2020).

${ }^{37}$ H. J. C. Berendsen, J. R. Grigera, and T. P. Straatsma, "The missing term in effective pair potentials," J. Phys. Chem. 91, 6269-6271 (1987).

${ }^{38}$ L. X. Dang, J. Am. Chem. Soc. 117, 6954 (1995).

${ }^{39}$ A. Berg, C. Peter, and K. Johnston, J. Chem. Theory Comput. 13, 5610 (2017). 